\title{
Breast Apocrine Adenosis
}

National Cancer Institute

\section{Source}

National Cancer Institute. Breast Apocrine Adenosis. NCI Thesaurus. Code C5198.

Breast adenosis characterized by the presence of extensive apocrine metaplasia. 